\title{
ON THE CONVERGENCE OF THE CALABI FLOW
}

\author{
WEIYONG HE
}

(Communicated by Lei $\mathrm{Ni}$ )

\begin{abstract}
Let $\left(M,\left[\omega_{0}\right], J\right)$ be a compact Kähler manifold without holomorphic vector field. Suppose $\omega_{0}$ is (the unique) constant scalar curvature metric. We show that the Calabi flow with any smooth initial metric converges to the constant scalar curvature metric $\omega_{0}$ with the assumption that Ricci curvature stays uniformly bounded.
\end{abstract}

\section{INTRODUCTION}

Let $\left(M,\left[\omega_{0}\right], J\right)$ be a compact Kähler manifold. The space of Kähler potentials is given by

$$
\mathcal{H}=\left\{\phi \in C^{\infty}: \omega=\omega_{0}+\sqrt{-1} \partial \bar{\partial} \phi>0\right\} .
$$

The Calabi flow is defined by E. Calabi in his seminal paper [1] as follows:

$$
\frac{\partial \phi}{\partial t}=R_{\phi}-\underline{R}
$$

where $R_{\phi}$ denotes the scalar curvature of $\omega_{\phi}$ and $\underline{R}$ is the average of scalar curvature, a topological constant depending only on $\left(M,\left[\omega_{0}\right], J\right)$.

It is a natural equation to seek a canonical representative in a fixed Kähler class (called extremal metric in [1]), which includes the Kähler metrics with constant scalar curvature as a special case. The Calabi flow is the gradient flow of Mabuchi's $K$-energy and is also a reduced gradient flow for the Calabi energy. One of the most challenging problems about the Calabi flow is whether it exists for all time or not. $\mathrm{X}$. X. Chen has made an ambitious conjecture as follows.

Conjecture 1 (Chen). The Calabi flow exists for all time for any initial potential in $\mathcal{H}$.

Donaldson [10] gives a conjectural picture of the asymptotic behavior of the Calabi flow and relates it to the stability conjecture regarding the existence of constant scalar curvature metric. In particular, it is expected that when there exists a constant scalar curvature metric in $\left(M,\left[\omega_{0}\right], J\right)$, the Calabi flow exists for all time and converges to a metric with constant scalar curvature (it is also called Donaldson's conjecture in the literature). Note that constant scalar metrics (more generally extremal metrics) in $\left(M,\left[\omega_{0}\right], J\right)$ is unique up to automorphism of $(M, J)$ (see Chen [3], Donaldson [9, Mabuchi [13], and finally for the most general case Chen-Tian [6, 7]). The longtime existence problem is still largely open (complex dimension is 2 or higher) except for a few special cases. In the present paper, we assume the following.

Received by the editors June 17, 2013 and, in revised form, July 19, 2013.

2010 Mathematics Subject Classification. Primary 53C55; Secondary 35K55. 
Assumption. Suppose the Calabi flow with any initial potential $\phi$ exists for all time with a uniform Ricci bound (for example, we assume a bound like $|\operatorname{Ric}(t)| \leq$ $C\left(\omega, \omega_{\phi},|\phi|_{C^{4}}\right)$ depending only on initial data and background geometry, but not on time). Note that by a result joint with X. X. Chen [5], the assumption on Ricci curvature actually implies the longtime existence.

The main result in this paper is to prove the following.

Theorem 2. With the assumption above, if there is a constant scalar curvature metric in $\left(M,\left[\omega_{0}\right], J\right)$ and there is no holomorphic vector field on $(M, J)$, then the Calabi flow converges to a constant scalar curvature metric for any initial metric in $\left(M,\left[\omega_{0}\right], J\right)$.

Remark 1.1. The main motivation of the result is that Chen's Conjecture 1 should imply Donaldson's conjecture that the Calabi flow converges to a constant scalar curvature metric when assuming such a metric exists. The Ricci curvature assumption is only technical in the proof. It will be interesting to drop this assumption.

\section{Evolution variational inequality along the Calabi flow}

In this section we prove a version of evolution variational inequality along the Calabi flow.

Proposition 2.1. Let $\phi_{t}$ be a smooth solution of the Calabi flow and $\psi \in \mathcal{H}$ be any point (not on $\left.\phi_{t}\right)$. Then

$$
K(\psi)-K\left(\phi_{t}\right) \geq d\left(\psi, \phi_{t}\right) \frac{d}{d t} d\left(\psi, \phi_{t}\right) .
$$

As a consequence, we have

$$
2 s\left(K(\psi)-K\left(\phi_{t+s}\right)\right) \geq d^{2}\left(\psi, \phi_{t+s}\right)-d^{2}\left(\psi, \phi_{t}\right),
$$

where $d$ is a natural distance function on $\mathcal{H}$ and it will be reviewed later.

Evolution variational inequality is well known for the gradient flow of convex functionals on Hilbert spaces. In two recent preprints, J. Streets [16, 17] constructed some kind of weak solution of the Calabi flow (with longtime existence) in the frame work of $K$-energy minimizing movements (see Mayer [14, for example, for some general results on minimizing movement of convex functionals on NPC spaces and Streets [16, 17] for $K$-energy minimizing movement). In particular he proved (2.2) and that $K$-energy minimizing movements minimizes $K$-energy and the Calabi energy asymptotically for any initial data. As a consequence, the Calabi flow minimizes $K$-energy and the Calabi energy when it is assumed to exist for all time (he does not assume the full Chen's conjecture, namely the longtime existence for all initial smooth metric).

We shall give an alternative proof of the evolution inequality (2.1) and (2.2) is a direct consequence. Our proof does not rely on the framework of minimizing movement. Actually it is rather straightforward given the results of Chen [3, 4] on the geometric structure of $\mathcal{H}$ and weak convexity of $K$-energy. One motivation for this proof is to understand the similar picture for Kähler-Ricci flow on Fano manifolds. In particular, we are interested in the following question. 
Question 3. Suppose $\left(M,\left[\omega_{0}\right], J\right)$ is a Fano manifold and suppose $\mathcal{F}$ functional is bounded below. Does the Kähler-Ricci flow minimize the $\mathcal{F}$ functional? In particular, we would like to know whether the Kähler-Ricci flow minimizes

$$
H(\omega)=\int_{M} h e^{h} \omega^{n},
$$

where $h$ is the normalized Ricci potential such that $\operatorname{Ric}(\omega)-\omega=\sqrt{-1} \partial \bar{\partial} h$ and

$$
\int_{M} e^{h} \omega^{n}=\int_{M} \omega^{n}
$$

We first recall the metric structure on $\mathcal{H}$, the space of Kähler potentials. Mabuchi [12] introduced a Riemannian metric on $\mathcal{H}$ and he also proved that this is formally a symmetric space with nonpositive curvature (see [8, 15] also). Chen [3] proved that $\mathcal{H}$ is actually a metric space and it is convex by $C^{1,1}$ geodesics (potential with bounded mixed derivative), by partially confirming conjectures of Donaldson [8], in which he set up an ambitious program which tightens up the existence of constant scalar curvature with the geometry of $\mathcal{H}$.

Our proof of evolution variational inequality relies on two results of Chen [3, 4 ] and indeed it is a rather straightforward consequence of his results. The first result we need is the following weak convexity of $K$-energy proved in [4. Let $A(t), 0 \leq t \leq 1$ be a geodesic in $\mathcal{H}$ with two end points $A(0)=\phi, A(1)=\psi$. Then $K$-energy is weakly convex in the sense that

$$
K(\psi)-K(\phi) \geq\left.\frac{d}{d t} K(A(t))\right|_{t=0}=\int_{M} \dot{A}(0)\left(\underline{R}-R_{\phi}\right) \omega_{\phi}^{n} .
$$

The second result is about the derivative of distance function $d$ on $\mathcal{H}$, which is proved in [3]. Suppose $\phi=\phi(s)$ is a smooth curve $C$ on $\mathcal{H}$. Then for any $\psi \in \mathcal{H}$ not on the curve $C, d(\psi, \phi)$ is $C^{1}$ on $s$ and, in particular,

$$
\frac{d}{d s} d(\psi, \phi)=-d(\psi, \phi)^{-1} \int_{M} \dot{A}(0) \frac{\partial \phi}{\partial s} \omega_{\phi}^{n},
$$

where $A(t)$ is the geodesic with $A(0)=\psi(s), A(1)=\psi$.

Given (2.3) and (2.4), suppose $\phi(s)$ solves the Calabi flow. It follows that

$$
K(\psi)-K(\phi(s))-d(\psi, \phi(s)) \frac{d}{d s} d(\psi, \phi(s)) \geq \int_{M} \dot{A}(0)\left(\underline{R}-R_{\phi}+\frac{\partial \phi}{\partial s}\right) \omega_{\phi(s)}^{n}=0 .
$$

This proved (2.1), while (2.2) can be obtained directly by taking integration from $t$ to $t+s$, noting that $K$-energy is decreasing along the flow. As a corollary, it follows that

Corollary 2.1. For any initial potential $\phi_{0} \in \mathcal{H}$, if the Calabi flow has a longtime solution $\phi(t)$ such that $\phi(0)=\phi_{0}$, then

$$
\lim _{t \rightarrow \infty} K(\phi(t))=\inf _{\psi \in \mathcal{H}} K(\psi) .
$$

Proof. This is a direct consequence of results proved in 17. Since the proof is rather short, we repeat it here. Suppose otherwise; hence there exists $\delta>0$ and $\psi \in \mathcal{H}$ such that for any $t$ sufficiently large,

$$
K(\psi)-K(\phi(t)) \leq-\delta .
$$


In (2.2), let $t=0, s \rightarrow \infty$. We get

$$
-2 s \delta \geq-d\left(\psi, \phi_{0}\right)^{2} .
$$

Contradiction.

Suppose the Calabi flow $\phi(t)$ exists for all time with initial metric $\phi_{0}$. We denote

$$
A\left(\phi_{0}\right)=\lim _{t \rightarrow \infty} \mathcal{C}(\phi(t)) .
$$

Clearly the limit exists and $A\left(\phi_{0}\right)$ is a nonnegative number.

Corollary 2.2. Suppose $\phi(t)$ and $\psi(t)$ are two longtime solutions of the Calabi flow with initial metrics $\phi_{0}$ and $\psi_{0}$ respectively. Then

$$
A\left(\phi_{0}\right)=A\left(\psi_{0}\right) \text {. }
$$

In other words, assuming Conjecture 1, the Calabi flow minimizes the Calabi energy asymptotically.

Proof. This is also a direct consequence of results proved in [17. We can assume that the curves $\phi(t)$ and $\psi(t)$ have no intersection. Otherwise we can assume that $\psi_{0}=\phi(t)$ for some $t$ for example. In this case it is clear that $A\left(\phi_{0}\right)=A\left(\psi_{0}\right)$. Suppose we have $A\left(\phi_{0}\right)=A\left(\psi_{0}\right)-3 \delta$ for some $\delta>0$. Since the statement only concerns the asymptotic behavior of the Calabi energy along $\phi(t)$ and $\phi(t)$, we can assume that for any $t$ (otherwise let $t$ be sufficiently large),

$$
\mathcal{C}\left(\phi_{t}\right) \leq \mathcal{C}\left(\psi_{t}\right)-\delta
$$

Note that if $\phi(t)$ is a solution of Calabi flow, by (2.2), we have, for $\psi$ not on the curve $\phi(t)$,

$$
2 s(K(\psi)-K(\phi(t+s))) \geq d^{2}(\psi, \phi(t+s))-d^{2}(\psi, \phi(t)) .
$$

Take $s=1$, and $\psi=\psi(t)$. We have

$$
d^{2}(\psi(t), \phi(t))+2(K(\psi(t))-K(\phi(t)))+2(K(\phi(t))-K(\phi(t+1))) \geq 0 .
$$

By a result of Calabi-Chen, we know that

$$
d^{2}(\psi(t), \phi(t)) \leq d^{2}\left(\phi_{0}, \psi_{0}\right) .
$$

Also we have

$$
K(\phi(t))-K(\phi(t+1))=\int_{t}^{t+1} \mathcal{C}(\phi(s)) d s \leq \mathcal{C}\left(\phi_{0}\right) .
$$

On the other hand,

and

$$
K\left(\psi_{0}\right)-K(\psi(t))=\int_{0}^{t} \mathcal{C}(\phi(s)) d s
$$

It follows that

$$
K\left(\phi_{0}\right)-K(\phi(t))=\int_{0}^{t} \mathcal{C}(\psi(s)) d s
$$

$$
K(\psi(t))-K(\phi(t))=\int_{0}^{t}(\mathcal{C}(\phi(s))-\mathcal{C}(\psi(s))) d s+K\left(\psi_{0}\right)-K\left(\phi_{0}\right) .
$$

By (2.5), we have

$$
K(\psi(t))-K(\phi(t)) \leq-\delta t+C .
$$

This contradicts (2.7) when $t$ is large enough. 
Remark 2.1. The results in this section are mostly proved by J. Streets [16, 17] in the framework of $K$-energy minimizing movement; indeed his results work for the more general setting of $K$-energy minimizing movements which exists all time for any initial data. He also proved that $K$-energy minimizing movement coincides with the Calabi flow when the latter exists; hence his results are applicable, for example, without assuming Conjecture 1 Our main motivation in this section is to establish (2.1) along the Calabi flow using a direct approach. We hope this argument could give an approach to Question 3.

\section{The CONVERGence of the CAlabi Flow}

We prove our main theorem in this section. Tian-Zhu [18] proved the convergence of Kähler-Ricci flow to a Kähler-Ricci soliton using a continuity method to deal with the Kähler-Ricci flow (see [19] also). Our method mimics their argument using the continuity method and the level set of certain functional. In Kähler-Ricci flow they use Perelman's entropy and we use the Calabi energy here.

First we can establish a finite time stability result. Given a fixed background metric $\omega$, we define a set $B=B(\lambda, \Lambda, K, \omega)$ as follows:

$$
B=\left\{\phi \in \mathcal{H}: \lambda \omega \leq \omega_{\phi} \leq \Lambda \omega,\|\phi\|_{C^{3, \alpha}} \leq K\right\} .
$$

In [5] (joint work with Chen), we proved a short time existence result of the Calabi flow with smoothing property and smooth dependence of initial data (see Theorem 3.2 in [5]) and the finite time stability is a consequence.

Proposition 3.1. Suppose the Calabi flow exists in the maximal time interval $[0, T)$ with initial potential $\phi_{0}$. Then for any $0<T_{0}<T$, there exists $\epsilon_{0}=\epsilon_{0}\left(\phi_{0}, T_{0}, \omega\right)$ such that for any potential $\left|\psi-\phi_{0}\right|_{C^{5}} \leq \epsilon_{0}$, the Calabi flow with initial potential $\psi$ exists in $\left[0, T_{0}\right]$ and

$$
\left|\psi\left(T_{0}\right)-\phi_{0}\left(T_{0}\right)\right|_{C^{5}} \leq C=C\left(\epsilon_{0}, \phi_{0}, T_{0}, \omega\right),
$$

where $C \rightarrow 0$ when $\epsilon_{0} \rightarrow 0$.

Proof. For any $T_{0}$ fixed, we know that $\phi_{0}(t)$ is a smooth path in $\mathcal{H}$ for $t \in\left[0, T_{0}\right]$. We can then pick up uniform constants $\lambda, \Lambda, K$ depending on $\phi_{0}, T_{0}$ such that $\phi_{0}(t) \in B=B(\lambda, \Lambda, K, \omega)$. We shall also assume that there exist a small number $\delta$ such that if

then $\psi \in B$.

$$
\min _{t \in\left[0, T_{0}\right]}\left|\psi-\phi_{0}(t)\right|_{C^{3, \alpha}} \leq \delta
$$

By Theorem 3.2 in [5], we know that for any potential $\psi \in B$, there exists uniform constants $t_{0}, \epsilon_{0}$ and $C_{0}$ depending only on $\lambda, \Lambda, K$ and $\omega$ such that the Calabi flow solution $\psi(t)$ exists for $\left[0, t_{0}\right]$; moreover, if

$$
\left|\psi-\phi_{0}\right|_{C^{3, \alpha}} \leq \epsilon_{0},
$$

then for any $t \in\left(0, t_{0}\right]$,

$$
\begin{aligned}
& \left|\psi(t)-\phi_{0}(t)\right|_{C^{3, \alpha}} \leq C_{0}\left|\psi-\phi_{0}\right|_{C^{3, \alpha}} \\
& \left|\psi(t)-\phi_{0}(t)\right|_{C^{4, \alpha}} \leq C_{0} t^{-1 / 4}\left|\psi-\phi_{0}\right|_{C^{3, \alpha}} .
\end{aligned}
$$

The smooth dependence part (3.1) is only emphasized around a constant scalar curvature metric in Theorem 3.2 of [5] (it is mainly used in the paper to prove the stability of a constant scalar curvature metric along the Calabi flow), but it holds 
around any smooth metric $\omega_{\phi_{0}}$ (it even holds for the initial metric in $C^{2, \alpha}$; see Theorem 3.1 in [5] and Theorem 2.1 in [11]). We shall also assume $\epsilon_{0} \leq \delta$. Let $k$ be the least integer such that $k \geq T_{0} / t_{0}$. Take $\epsilon$ small enough such that $\left(C_{0}\right)^{k} \epsilon<\epsilon_{0}$. Then for any $\psi \in B$ satisfying

$$
\left|\psi-\phi_{0}\right|_{C^{5}} \leq \epsilon
$$

we get that

$$
\left|\psi\left(t_{0}\right)-\phi_{0}\left(t_{0}\right)\right|_{C^{3, \alpha}} \leq C_{0} \epsilon \text {. }
$$

Clearly $\psi\left(t_{0}\right)$ and $\phi_{0}\left(t_{0}\right)$ are both in $B$. We can then apply Theorem 3.2 in [5] to the initial potential $\phi\left(t_{0}\right)$ and $\psi\left(t_{0}\right)$ to get a Calabi flow solution $\psi(t)$ in $\left[t_{0}, 2 t_{0}\right]$. We can repeat this argument to get a solution $\psi(t)$ in $\left[0, k t_{0}\right]$ which contains $\left[0, T_{0}\right]$. (We emphasize that there is no contradiction since $t_{0}$ depends on $T_{0}$ and we know that $T_{0} \leq k t_{0}<T$; we cannot repeat the argument for $t>T_{0}$.) It then follows from (3.1) that, for any $t \in\left[0, T_{0}\right]$,

$$
\left|\psi(t)-\phi_{0}(t)\right|_{C^{3, \alpha}} \leq\left(C_{0}\right)^{k} \epsilon<\epsilon_{0} .
$$

By the smoothing property, we can get that

$$
\left|\psi\left(T_{0}\right)-\phi_{0}\left(T_{0}\right)\right|_{C^{5}} \leq C\left(\epsilon_{0}, \lambda, \Lambda, K, T_{0}, \omega\right),
$$

where $C$ goes to zero when $\epsilon_{0}$ goes to zero.

Remark 3.1. With the assumption on Ricci curvature, the above finite time stability is instant with the compactness theorem in 5 .

Now we are in the position to prove Theorem 2

Proof. Note that we assume there is no holomorphic vector field. Let $\omega_{0}$ be the unique constant scalar curvature metric in $\left(M,\left[\omega_{0}\right]\right)$ (uniqueness is proved by ChenTian). Let $\omega_{\phi}=\omega_{0}+\sqrt{-1} \partial \bar{\partial} \phi$ (we assume a normalization condition $I(\phi)=0$ ). Denote

$$
G=\left\{\phi \in \mathcal{H}:|\phi(t)|_{C^{5}} \rightarrow 0, t \rightarrow \infty\right\}
$$

to be the initial potentials such that $\phi(t)$ solves the Calabi flow equation and converges to zero (hence $\omega_{\phi(t)}$ converges to $\omega_{0}$ ). Clearly $0 \in G$ and let $\phi \in \mathcal{H}$. Let $\phi_{s}, 0 \leq s \leq 1$ be a smooth path in $\mathcal{H}$ such that $\phi_{0}=0, \phi_{1}=\phi$ (we can choose $\phi_{s}=s \phi$ say). Denote the corresponding Calabi flow solution as $\phi_{s}(t)$. We want to show that $\phi_{s}(t) \in G$. Now let

$$
S=\left\{s \in[0,1]: \phi_{s} \in G\right\} .
$$

We want to prove $S=[0,1]$. By the method of continuity, we need to show $S$ is open and closed. This completes the proof of Theorem 2, given the two propositions below.

First we prove the openness of $S$. The openness follows essentially from the finite time stability and the stability theorem around the constant scalar curvature metric proved in [5]. In particular, we have

Proposition 3.2. Suppose $s \in S$. Then for $\delta$ small enough $(s-\delta, s+\delta) \cap[0,1] \in S$.

Proof. Since $\omega_{\phi_{s}(t)}$ converges to $\omega_{0}$; hence $\left|\phi_{s}(t)\right|_{C^{5}} \rightarrow 0$ when $t$ is sufficiently large. By stability of constant scalar curvature metric along the Calabi flow, we 
can assume that there exists $\epsilon=\epsilon\left(\omega_{0}\right)$ such that

$$
|\phi|_{C^{5}} \leq 2 \epsilon ;
$$

then $\phi \in G$. Let $T$ be sufficiently large and assume that $\left|\phi_{s}(T)\right|_{C^{5}} \leq \epsilon$. Fix $T$, and by Proposition 3.1. there exists $\epsilon_{1}$ small enough such that $\left|\psi-\phi_{s}\right|_{C^{5}} \leq \epsilon_{1}$. Then

$$
|\phi(T)-\psi(T)|_{C^{5}} \leq c\left(\epsilon_{1}, \phi_{s}, T\right) .
$$

We choose $\epsilon_{1}$ small enough such that $c\left(\epsilon_{1}, \phi_{s}, T\right) \leq \epsilon$. Then

$$
|\psi(T)| \leq 2 \epsilon \text {. }
$$

Applying Theorem 4.1 in [5] to $\psi(T)$, we get that $\psi(T) \in G$. It then follows that there exists $\epsilon_{1}$ such that $\psi \in G$ provided

$$
\left|\psi-\phi_{s}\right|_{C^{5}} \leq \epsilon_{1}
$$

Hence if $s \in S$, we can choose $\delta$ small enough such that $\phi_{r} \in G$ for $r \in(s-\delta, s+$ $\delta) \cap[0,1]$.

Next we show $S$ is closed. To show $S$ is closed, it suffices to show that if $[0,1) \subset S$, then $1 \in S$. Actually we want to show if $[0,1) \subset S$, then the following uniform estimates for $\phi_{s}(t)$ for any $s \in[0,1)$ holds.

Proposition 3.3. For any $\delta>0$ and $s \in[0,1)$, there exists $T>0$ such that for any $t \geq T$,

$$
\left|\phi_{s}(t)\right|_{C^{5}} \leq \delta .
$$

Proof. We argue by contradiction. Suppose otherwise; hence then there exists a sequence of $s_{i} \rightarrow 1$ and $t_{i} \rightarrow \infty$ such that

$$
\left|\phi_{s_{i}}\left(t_{i}\right)\right|_{C^{5}} \geq \delta
$$

for some $\delta>0$, since for any $s_{i} \in[0,1),\left|\phi_{s_{i}}(t)\right|_{C^{5}} \rightarrow 0$ when $t \rightarrow \infty$. We can then assume $t_{i}$ satisfies the following:

$$
\left|\phi_{s_{i}}\left(t_{i}\right)\right|_{C^{5}}=\delta \geq\left|\phi_{s_{i}}(t)\right|_{C^{5}}, t \geq t_{i} .
$$

Let $\psi_{i}(t)=\phi_{s_{i}}\left(t_{i}+t\right)$ for $t \in[-1,1]$. Then we know that $\psi_{i}(t)$ is a sequence of solutions of Calabi flow on $\left(M,\left[\omega_{0}\right], J\right)$ in $[0,1]$ such that

$$
\left|\psi_{i}(t)\right|_{C^{5}} \leq \delta .
$$

By the compactness theorem, we get that $\psi_{i}(t)$ converges to $\psi_{\infty}(t)$ such that $\psi_{\infty}(t)$ is still a solution of the Calabi flow in $[0,1]$. Note that, at the moment, the convergence

$$
\psi_{i}(t) \rightarrow \psi_{\infty}(t)
$$

is only in $C^{4, \alpha}$ for any $\alpha \in(0,1)$. Now we claim that $\phi_{\infty}(t) \equiv 0$ for $t \in[0,1]$. When $s=1$, let $\phi_{1}(t)=\phi(t)$ be the solution of the Calabi flow. By Corollary 2.2, we know that for any solution of the Calabi flow $\phi(t)$, the Calabi energy has the same energy level at infinity. Hence $\mathcal{C}(\phi(t)) \rightarrow 0$ when $t \rightarrow \infty$. For any $\epsilon>0$, we can then find $t_{0}$ sufficiently large such that

$$
\mathcal{C}\left(\phi\left(t_{0}\right)\right)<\epsilon / 2 \text {. }
$$

By Proposition 3.1, we get that for $i$ sufficiently large,

$$
\mathcal{C}\left(\phi_{s_{i}}\left(t_{0}\right)\right)<\epsilon \text {. }
$$


It then follows that for $i$ sufficiently large and $t \in[0,1]$,

$$
\mathcal{C}\left(\psi_{i}(t)\right)=\mathcal{C}\left(\phi_{s_{i}}\left(t_{i}+t\right)\right)<\epsilon .
$$

We can then conclude that for any $t \in[0,1]$,

$$
\mathcal{C}\left(\psi_{\infty}(t)\right)=0 \text {. }
$$

Hence $\psi_{\infty}(t)$ are all potentials of the constant scalar curvature metric. By the uniqueness theorem, we know that $\psi_{\infty}(t) \equiv 0$ (we assume that there is no holomorphic vector field). In particular we know that

$$
\psi_{i}(t) \rightarrow 0
$$

in $C^{4, \alpha}$ for $i$ sufficiently large. To get a contradiction, we use the assumption that the Ricci curvature is uniformly bounded for all $i$. Consider $\psi_{i}(t)$ in $[-1,1]$. Then we know that (since the Ricci curvature, hence the scalar curvature is uniformly bounded),

$$
\left|\partial_{t} \psi_{i}(t)\right| \leq C
$$

Hence it follows that $\psi_{i}(t)$ is uniformly bounded in $[-1,1]\left(\psi_{i}(0)\right.$ is uniformly small in $\left.C^{5}\right)$. By the compactness theorem in [5], we know that $\psi_{i}(t)$ is uniformly bounded in $[-1,1]$ in $C^{3, \alpha}$. Now applying the smoothing property of the Calabi flow to $\psi_{i}(t)$ in $t \in[-1,1]$ for $i$ sufficiently large, it follows that for any $t \geq 0$,

$$
\left|\psi_{i}(t)\right|_{C^{k}} \leq C(k, t) \text {. }
$$

In other words, $\psi_{i}(t)$ converges to 0 in $C^{\infty}$ for any $t \geq 0$, when $i \rightarrow \infty$. This clearly contradicts the fact that $\left|\psi_{i}(0)\right|_{C^{5}}=\delta$.

Remark 3.2. The Ricci curvature assumption is only used to get an improved regularity at time $t=0$ for $\psi_{i}(t)$. Such an assumption is rather technical; we hope we can overcome the difficulty to drop the Ricci curvature assumption in the future. The assumption on nonexistence of holomorphic vector fields is rather superfluous and the similar strategy should also apply to extremal metrics. We will leave these problems for future study since Ricci curvature assumption seems to be more serious.

\section{ACKNowledgements}

The author thanks Bing Wang and Song Sun for valuable discussions and also thanks X. X. Chen for encouragement. The author is also grateful to J. Streets for sending him his recent preprint [17] which inspired the consideration in Section 2. The author also thanks the referee for valuable comments and suggestions. The author was partially supported by an NSF grant.

\section{REFERENCES}

[1] Eugenio Calabi, Extremal Kähler metrics, Seminar on Differential Geometry, Ann. of Math. Stud., vol. 102, Princeton Univ. Press, Princeton, N.J., 1982, pp. 259-290. MR645743 (83i:53088)

[2] E. Calabi and X. X. Chen, The space of Kähler metrics. II, J. Differential Geom. 61 (2002), no. 2, 173-193. MR1969662 (2004i:32039)

[3] Xiuxiong Chen, The space of Kähler metrics, J. Differential Geom. 56 (2000), no. 2, 189-234. MR:1863016 (2003b:32031)

[4] Xiuxiong Chen, Space of Kähler metrics. III. On the lower bound of the Calabi energy and geodesic distance, Invent. Math. 175 (2009), no. 3, 453-503, DOI 10.1007/s00222-008-0153-7. MR2471594(2010b:32033) 
[5] X. X. Chen and W. Y. He, On the Calabi flow, Amer. J. Math. 130 (2008), no. 2, 539-570, DOI 10.1353/ajm.2008.0018. MR2405167 (2009c:53086)

[6] Xiuxiong Chen and Gang Tian, Partial regularity for homogeneous complex Monge-Ampere equations (English, with English and French summaries), C. R. Math. Acad. Sci. Paris 340 (2005), no. 5, 337-340, DOI 10.1016/j.crma.2004.11.024. MR2127106 (2006d:32053)

[7] X. X. Chen and G. Tian, Geometry of Kähler metrics and foliations by holomorphic discs, Publ. Math. Inst. Hautes Études Sci. 107 (2008), 1-107, DOI 10.1007/s10240-008-0013-4. MR2434691 (2009g:32048)

[8] S. K. Donaldson, Symmetric spaces, Kähler geometry and Hamiltonian dynamics, Northern California Symplectic Geometry Seminar, Amer. Math. Soc. Transl. Ser. 2, vol. 196, Amer. Math. Soc., Providence, RI, 1999, pp. 13-33. MR1736211 (2002b:58008)

[9] S. K. Donaldson, Scalar curvature and projective embeddings. I, J. Differential Geom. 59 (2001), no. 3, 479-522. MR.1916953 (2003j:32030)

[10] Simon K. Donaldson, Conjectures in Kähler geometry, Strings and geometry, Clay Math. Proc., vol. 3, Amer. Math. Soc., Providence, RI, 2004, pp. 71-78. MR2103718 (2005i:32024)

[11] Weiyong He, Local solution and extension to the Calabi flow, J. Geom. Anal. 23 (2013), no. 1, 270-282, DOI 10.1007/s12220-011-9247-3. MR3010280

[12] Toshiki Mabuchi, Some symplectic geometry on compact Kähler manifolds. I, Osaka J. Math. 24 (1987), no. 2, 227-252. MR909015 (88m:53126)

[13] Toshiki Mabuchi, Uniqueness of extremal Kähler metrics for an integral Kähler class, Internat. J. Math. 15 (2004), no. 6, 531-546, DOI 10.1142/S0129167X04002429. MR2078878 (2005c:32029)

[14] Uwe F. Mayer, Gradient flows on nonpositively curved metric spaces and harmonic maps, Comm. Anal. Geom. 6 (1998), no. 2, 199-253. MR.1651416 (99m:58067)

[15] Stephen Semmes, Complex Monge-Ampère and symplectic manifolds, Amer. J. Math. 114 (1992), no. 3, 495-550, DOI 10.2307/2374768. MR.1165352 (94h:32022)

[16] J. Streets, Long time existence of Minimizing Movement solutions of Calabi flow, arxiv.org/abs/1208.2718.

[17] J. Streets, The consistence and convergence of K-energy minimizing movement, http://arxiv.org/abs/1301.3948.

[18] Gang Tian and Xiaohua Zhu, Convergence of the Kähler-Ricci flow on Fano manifolds, J. Reine Angew. Math. 678 (2013), 223-245. MR3056108

[19] G. Tian, S.J Zhang, Z.L Zhang, X.H. Zhu, Supremum of Perelman's entropy and Kähler-Ricci flow on a Fano manifold, arxiv.org/abs/1107.4018.

Department of Mathematics, University of Oregon, Eugene, Oregon 97403

E-mail address: whe@uoregon.edu 